\title{
Indexes of Cardiac and LAD Irradiation in Conventional 2-Dimensional (SAD) and 3-Dimensional Conformal Radiotherapy Techniques: A Dosimetrical Comparison
}

\author{
Emami Hamid ${ }^{1 *}$, Akhavan Ali ${ }^{1}$, Sharhani Refagh ${ }^{1}$, Karami Mehdi ${ }^{1}$, Amouheidari Alireza $^{2}$, Raoufi Seyedamir ${ }^{3}$, Shams Ali ${ }^{1}$ and $^{\text {Monadi Shahram }}{ }^{1}$ \\ ${ }^{1}$ Department of Radiation Oncology, Seyed Al-Shohada Charity Hospital, Isfahan, Iran \\ ${ }^{2}$ Department of Radiation Oncology, Isfahan Milad Hospital, Isfahan, Iran \\ ${ }^{3}$ Department of Cardiology, Seyed Al-Shohada Charity Hospital, Isfahan, Iran \\ *Corresponding author: Emami Hamid, Department of Radiation Oncology, Seyed Al-Shohada Charity Hospital, Isfahan, Iran, Tel: 9809368668279; E-mail: \\ h_emami@med.mui.ac.ir
}

Received date: June 29, 2017; Accepted date: July 27, 2017; Published date: July 31, 2017

Copyright: @ 2017 Emami H, et al. This is an open-access article distributed under the terms of the Creative Commons Attribution License, which permits unrestricted use, distribution, and reproduction in any medium, provided the original author and source are credited.

\begin{abstract}
Radiation therapy in breast cancer patients has been shown to reduce local recurrence and improve survival rates. Nevertheless, recent concerns have been raised regarding an increase in cardiac mortality, especially in patients who had been treated with radiation for left-sided breast tumors. Hence, the aim of this study is to estimate doses of irradiation to the heart, LAD coronary artery, and lungs in 2D (SAD) and 3D conformal radio therapy techniques, and to compare the two.
\end{abstract}

Out of 32, 18 to 75 years old females with a diagnosis of non-metastatic breast cancer, who referred from surgery department, went through irradiation in the conventional 2D (SAD) and then 3DCRT using multi-slice CT scans to contour vital organ. Nearly all of the dosimetrical indexes were higher in 3DCRT method.

This shows that 2D technique lacks a decent scale to measure cardiac toxicity since its mid-plane CT slice did not give a reliable assessment of cardiac irradiation.

Keywords: Breast cancer; Dosimetry; Cardiac toxicity; study carried out by Darby et al. it is shown that with every $1 \mathrm{~Gy}$ Radiotherapy; 3DCRT increase in average irradiation dosage, there would a $7.4 \%$ increase in coronary accidents [8]. Also recent studies have indicated that these risks are exacerbated particularly when radiation is applied as an adjuvant program beside chemotherapy $[11,12]$. Diagnosing cardiac complications is quite a challenge, since they will not be distinguishable until at least 10 years from the radiation $[13,14]$. This latent manifestation of cardiovascular diseases is of great importance, given the fact that survival rate for breast cancer patients is improving, and breast cancer patients have remarkable life expectancy after completing treatment plans. This emphasizes on the importance of reducing the probability in cardiac mortality.

Evaluation of the potential cardiac toxicity of radiotherapy can lead us to utilization of more advanced radiation facilities. However, there is a conundrum on the advantage/disadvantage for practitioner to decide between choosing the most competent treatment plan in order for the least recurrence rate possible to happen, and choosing the lowest effective treatment in order to reduce the later complications as much as possible. Only few studies focusing on comparison of different external beam radiotherapy techniques were found on the databases. We have started to treat breast cancer patients in Omid Hospital, Isfahan, Iran, with 3DCRT technique for almost a year. Hence, the aim of this study is to estimate doses of irradiation to the heart, LAD coronary artery, and lungs in $2 \mathrm{D}(\mathrm{SAD})$ and $3 \mathrm{D}$ conformal radio therapy techniques, and to compare the two. Un breast cancer patients treated with radiotherapy in the United States have shown that mortality rates in cases with left-sided breast tumors is more than in those with right-sided breast tumors. These evidences strongly support the fact that cardiac exposure is a serious issue in left-sided breast cancer patients after radiotherapy. In a 
Citation: Hamid E, Ali A, Refagh S, Mehdi K, Alireza A, et al. (2017) Indexes of Cardiac and LAD Irradiation in Conventional 2-Dimensional (SAD) and 3-Dimensional Conformal Radiotherapy Techniques: A Dosimetrical Comparison. J Nucl Med Radiat Ther 8: 337. doi: $10.4172 / 2155-9619.1000337$

Page 2 of 6

\section{Method and Materials}

\section{Subjects}

Thirty-two 18 to 75-year-old females with a diagnosis of nonmetastatic breast cancer, who referred from surgery department after either breast conservative surgery or modified radical mastectomy, Omid Hospital, Isfahan, Iran, between 2015 and 2016, were included in this retrospective study. Our sampling method was simple nonprobability, and patients who had a history of previous radiation therapy, lupus erythromatosis, or sclerodermia were excluded from the study.

\section{Method}

After CT simulation, 2D (SAD) and 3DCRT treatment planning were made for each patient. For each treatment plan, dose-volume histograms (DVHs) for the heart and for the LAD, with a $1-\mathrm{cm}$ radial margin, were generated. We also measured treatment volumes and other dosimetrical indexes (to be furtherly determined).

An important step in the process of treatment planning in is computer planning and dose calculations, using CT scans that were not contrast-enhanced. A CT scan was obtained from upper margin of cricoid cartilage onto lower limit of the lungs with 1.5-millimeter slice thickness. Patients' left arms were elevated as much as possible since the CT machine was not big core and we were not able to elevate both arms of the patients. CT scan was performed in a supine position on each patient in 16-slice Siemense Somatom Scope. Once the CT scan is obtained, the radiation oncologist uses a treatment planning computer to personally outline all areas of interest including specific targets of treatment, as well as any normal vital structures which may be irradiated and influenced. This delineation and contouring was done under the observation of an experienced radiologist and in accordance to RTOG atlas. Once all areas were designated, a 3-dimensional reconstruction was performed for each subject. Appropriate radiation fields were then added with appropriate blocking to exclude as much normal tissue as possible. The doses were then opted as per the practitioner's treatment prescription. The prescribed dose was 5000cGy in 25 fractions in both techniques. No additional boost was given to the tumor bed. All patients were positioned on a breast board with the sternum horizontal to the treatment couch and ipsilateral arm above the head. The tangential field borders were determined clinically by the attending clinician and marked with radio-opaque wires. In 2D techniques, one radio-opaque wire is placed in mid-line, and another wire is placed lateral to the breast for the purpose of simulation. It was decided to limit the number of slices requested for each patient to two One slice at the level of the maximum heart (determined either using fluoroscopy or from a scannogram depending on the equipment) and the other the treatment field central axis. The medial border was $1 \mathrm{~cm}$ ipsilateral to the mid-line, the superior border was the sternal notch, the inferior border was $1 \mathrm{~cm}$ below the infra- mammary fold, and the lateral border was $1 \mathrm{~cm}$ outside the lateral palpable border of the breast (or the mid-axillary line for mastectomized patients). These margins were helpful for uncertainty in identification of arterial position, respiratory movement, and for the beating movement of the heart. The cardiac dose distributions were calculated with the full threedimensional CT set. The patient surface was defined by automated density gradient tracking. The organs at risk that were assessed were the heart, left ascending coronary artery, and left lung. The heart and left anterior descending (LAD) coronary artery were outlined for all patients. Therefore, on some CT images it was not possible to visualize the coronary arteries directly, and their location was inferred using visible, reliable, cardiac landmarks as follows: The location of the LAD coronary artery was identified using the course of the anterior interventricular groove. The contours were reviewed and modified, where appropriate, by one of the authors (radiologist). Threedimensional alignment was built up on the basis of multi-slice CT scan planes and with the help of treatment planning software (TPS) and advanced shielding. The TPS dosimetry audit was performed in accordance to IAEA methodology for dosimetry in 3DCRT; TECDOC 1583 [15]. After 2D and 3D simulation for each subject, whole breast and chest wall contouring, and for patients with axillary lymph node involvement (T3), axillary and supraclavicular lymph node contouring was done if necessary. Note that we did not treat internal mammary lymph nodes. The calculation of the dose inside the open part of the beam (6MV) was within $1 \%$ for normal tissue and within $4-5 \%$ for equivalent lungs tissue. However, dose calculation outside the open field in equivalent lungs tissue was within $1 \%$ for $6 \mathrm{MV}$ beams. For the purpose of evaluating the target volume, dosimetrical distributions were measured and margins of the target were defined by TiGRT software v. 1.0.10.573 (Linach co.). After optimizing the borders, beam angle, and other factors, we were able to observe the distribution of doses exclusively or all-together. In order to simulate 2-D therapy techniques, two guide wires were applied and fixed on each patient right before being sent to obtain a CT scan; one surrounding patient's breast, and one on the lateral side of her breast. CT scan was then obtained and 2D method was designed based on beams, shielding, and etc, using the same software. At this time dosimetrical distributions and dose-volume histograms (DVH) were available for us to observe. A DVH can not only give us the quantitative data on how much dose every volume unit absorbs, but also provides us with dosimetrical information of distribution in any anatomical structure, all summarized in a curve. The technical and practical aspects of implementing this technique in the clinic were then analyzed. We analyzed our data using SPSS v. 16.0.

Indexes: We aimed to draw a decent comparison between 2 dimensional (SAD)in which we only had the middle slice and 3dimensional technique in which we contour several organs at risk using multi-slice CTscan in several indexes and quantities which are defined as below; 3 organs in the radiation site which are unintentionally exposed to irradiation are as listed below:

- Heart: Main indexes of toxicity estimation:

Mean Dose (cGy)

\section{Maximum Dose (cGy)}

Maximal heart distance (MHD): the width of heart in the tangent fields at its maximal level in tangential fields referring to the heart contour in a digitally reconstructed radiograph (DRR). (mm)

V20: Volume proportion of the heart which receives 20Gy o higher dose of radiation $(\%)$

- Left Anterior Descending (LAD) coronary artery:

Mean dose

Maximum dose

LAD Volume: The volume of LAD artery with an extra margin of 1 $\mathrm{cm}$ which is considered as safe margin (cc)

- Lung: Main indexes by which we estimate the level of toxicity overloaded to the lung are: 
Citation: Hamid E, Ali A, Refagh S, Mehdi K, Alireza A, et al. (2017) Indexes of Cardiac and LAD Irradiation in Conventional 2-Dimensional (SAD) and 3-Dimensional Conformal Radiotherapy Techniques: A Dosimetrical Comparison. J Nucl Med Radiat Ther 8: 337. doi: $10.4172 / 2155-9619.1000337$

Page 3 of 6

Maximum Lung Distance (MLD): The maximum perpendicular distance from the posterior tangential field edge to the posterior part of the anterior chest wall $(\mathrm{mm})$

V20: Volume proportion of the lung receiving 20Gy or higher dose of radiation (\%)

V10: Volume proportion of the lung receiving 10Gy or higher dose of radiation (\%)

V5: Volume proportion of the lung receiving 5Gy or higher dose of radiation $(\%)$

Since clips were not applied on our patients, we were not certain if we are giving the needed boost to the right location, and also electron was not provided in our hospital, we gave every patient a standard radiation of $5000 \mathrm{cGy}$.

* Maximum distances (or depths) were measured in millimeters as the largest perpendicular distance between the anterior contours of the organs and the posterior tangential field edges.

\section{Results}

There were 32 patients with left-sided breast cancer with mean age of $51.9 \pm 56.9$ were included in this study. 20 patients had undergone Breast Conservative Treatment (BCT) and 12 had undergone Modified Radical Mastectomy (MRM). This difference in operation procedure was not significant $(p>0.05)$. Data was analyzed with paired t-test.
As it is shown in Table 1, our patients received an average dose of $627.9 \pm 319.87$ (cGy) to heart and $2536.75 \pm 1086.3$ (cGy) to LAD coronary artery in 2-dimensional method. Also they have been exposed to doses of $1079 \pm 425$ and $3487.4 \pm 769.6$ in their heart and LAD, in 3DCRT respectively. Average maximum doses given to our subjects in 2-dimensional and 3-dimensional methods were $5068 \pm$ 917.6(cGy) and 5423.8 \pm 346.9 (cGy) respectively for the heart, and $4751.5 \pm 965.2$ (cGy) and 5134.6 \pm 656 (cGy) for LAD, respectively.

As it can be seen in Figure 1, doses given to normal major organs in left thoracic area are mainly higher in 3D method. These differences, as inferred from the Figure, and seen in Table 1, are significant in nearly all four indexes with mean heart and mean LAD dose having highly significant differences. As given Figure 2, maximum lungs distance is remarkably higher in 3DCRT method, with a mean of $4.05 \pm 0.87$ as compared to $3.01 \pm 1.03$. Also maximum heart distance is remarkably lower in 3D method, with an average of $1.50 \pm 0.91$ as compared to $2.55 \pm 0.91$. These differences, as inferred from Table 1 and Figure 2, are both highly significant. As it can be seen in Figure 3, volume proportions of the major organs in left thoracic area which are exposed to irradiation are all higher in 3D method, with $69.28 \pm 16.79$ (\%) as compared to $49.07 \pm 25.57$ (\%) in LAD exposure volume. Volumes of heart and lung with 20Gy and volumes of lung with 5 and $10 \mathrm{cGy}$ radiation exposures are shown in Table 1 . The difference in all volume indexes in the present study is highly significant $(\mathrm{p}<0.001)$.

\begin{tabular}{|c|c|c|c|c|c|}
\hline Variable & Mean & Standard Deviation & MDâ & $\begin{array}{l}\text { Standard Deviation } \\
\text { (MD) }\end{array}$ & $\mathbf{P}$ \\
\hline Mean heart dose-2D & 627.9 & 319.87 & -451.18 & 321.77 & $<0.001$ \\
\hline Mean heart dose-3D & 1079.09 & 424.05 & & & \\
\hline Maximum heart dose-2D & 5068.03 & 917.63 & -355.81 & 930.33 & 0.038 \\
\hline Maximum heart dose-3D & 5423.84 & 346.96 & & & \\
\hline Mean LAD dose-2D & 2536.75 & 1086.31 & -950.65 & 909.51 & $<0.001$ \\
\hline Mean LAD dose-3D & 3487.4 & 769.64 & & & \\
\hline Maximum LAD dose-2D & 4751.59 & 965.22 & -383.06 & 1058.56 & 0.049 \\
\hline Maximum LAD dose-3D & 5134.65 & 656.08 & & & \\
\hline MHD1-2D & 2.55 & 0.91 & 1.04 & 0.9 & $<0.001$ \\
\hline MHD-3D & 1.5 & 0.91 & & & \\
\hline Volume of LAD-2D & 49.07 & 25.57 & -20.2 & 22.57 & $<0.001$ \\
\hline Volume of LAD-3D & 69.28 & 16.79 & & & \\
\hline V20 of heart-2D & 10.85 & 7.89 & -6.71 & 8.73 & $<0.001$ \\
\hline V20 of heart-3D & 17.56 & 8.54 & & & \\
\hline V20 of lung-2D & 13.21 & 6.94 & -10.17 & 5.29 & $<0.001$ \\
\hline V20 of lung-3D & 23.38 & 6.07 & & & \\
\hline V10 of lung-2D & 15.23 & 7.27 & -13.03 & 4.9 & $<0.001$ \\
\hline V10 of lung-3D & 28.26 & 6.92 & & & \\
\hline
\end{tabular}


Citation: Hamid E, Ali A, Refagh S, Mehdi K, Alireza A, et al. (2017) Indexes of Cardiac and LAD Irradiation in Conventional 2-Dimensional $(\mathrm{SAD})$ and 3-Dimensional Conformal Radiotherapy Techniques: A Dosimetrical Comparison. J Nucl Med Radiat Ther 8: 337. doi: $10.4172 / 2155-9619.1000337$

Page 4 of 6

\begin{tabular}{|l|l|l|l|l|l|}
\hline V5 of lung-2D & 18.84 & 7.69 & -19.98 & 9.03 & $<0.001$ \\
\hline V5 of lung-3D & 38.82 & 8.51 & & & \\
\hline MLD2-2D & 3.01 & 1.03 & -1.03 & 0.94 & $<0.001$ \\
\hline MLD-3D & 4.05 & 0.87 & & \\
\hline "Mean difference, ${ }^{1}$ Maximum heart distance, ${ }^{2}$ Maximum lungs distance
\end{tabular}

Table 1: Dosimetrical indexes in 2D (SAD) and 3DCRT techniques.

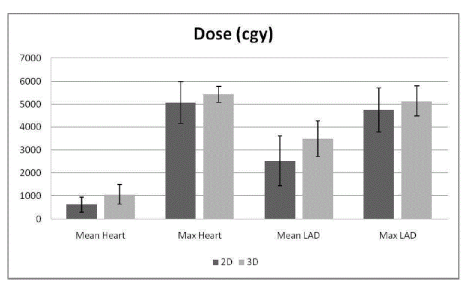

Figure 1: Average mean and average maximum dosimetry of irradiation in 2D and 3D treatment methods in heart and LAD.

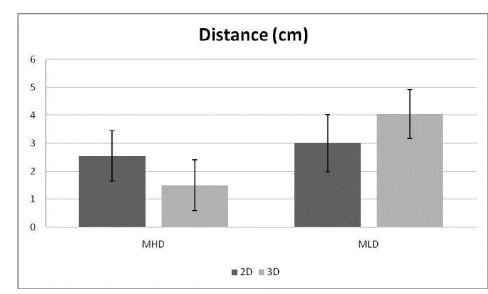

Figure 2: Maximum heart distance and maximum lung distance in $2 \mathrm{D}$ and $3 \mathrm{D}$ treatment methods.

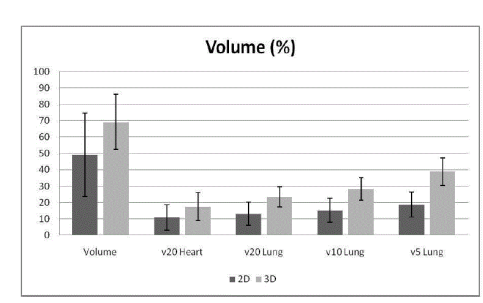

Figure 3: Volumes of organs undergoing a certain amount of irradiation in $2 \mathrm{D}$ and $3 \mathrm{D}$ treatment methods in heart, LAD and lung.

\section{Discussion}

In the present study, we accomplished a comparison of dosimetrical indexes, all well described in the methods section, in 3D conformal breast planning techniques and the conventional 2D (SAD) approach. In other words, we conducted this study to evaluate the dosimetric effect of introducing additional shielding to reduce heart and LAD coronary artery dose. This choice of techniques of ours was basically manipulated by the lack of availability in variable RT techniques, such as multi-leaf collimator, breath-holding method, or IMRT, since SAD method was the only available technique in our center by 2014 . In the conventional 2D method (SAD technique) patients' breast was irradiated only on the basis of anatomical landmarks and the estimated location of the tumor. As a result of this method, neither the breast and its tumor, nor the normal structures in that local area such, as the heart and LAD, were irradiated as much as more advanced and novel treatment methods like 3DCRT (Figures 4 and 5).

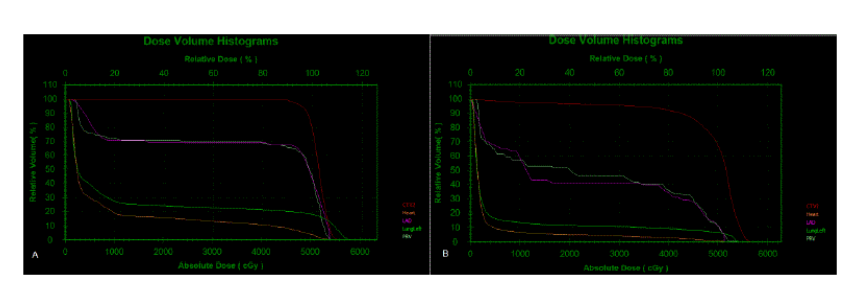

Figure 4: (A) Dose-volume histograms for a 3D breast irradiation plan. For each dose level on the horizontal axis the per cent volume of each organ receiving that dose level or more is plotted. The dose delivered to several structures is assessed. The breast should be covered by $90-110 \%$ of the prescription dose (here $48 \mathrm{~Gy}$ ). The dose to critical healthy organs should be limited. In this case, the left lung receives a dose of $20 \mathrm{~Gy}$ or more to $25 \%$ of its total volume. The volume of arch left anterior descending coronary artery (LAD) irradiated to $20 \mathrm{~Gy}$ is $70 \%$. (B) Dose-volume histogram calculated fo $2 \mathrm{D}$ breast irradiation. It is clear in this picture that $90 \%$ of the target has received $4200 \mathrm{cGy}$ and $95 \%$ has received 3600cGy.

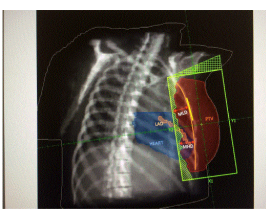

Figure 5: Demonstration of treatment parameters measured from patient 3 dimensional medial (beam's-eye view) treatment film. The maximum lungs distance (MLD), maximum heart distances (MHD) are shown. Major proportion of anterior section of the heart is located in tangential field of left breast radiation. With a more excessive shielding breast PTV in posterior part of the tangential field would be shielded also.

What mainly has come out as our findings is that in these 32 subjects, the irradiation dosimetry in 3DCRT was significantly higher 
Citation: Hamid E, Ali A, Refagh S, Mehdi K, Alireza A, et al. (2017) Indexes of Cardiac and LAD Irradiation in Conventional 2-Dimensional (SAD) and 3-Dimensional Conformal Radiotherapy Techniques: A Dosimetrical Comparison. J Nucl Med Radiat Ther 8: 337. doi: $10.4172 / 2155-9619.1000337$

Page 5 of 6

in almost all indexes, except for maximum heart distance. We would like to discuss this matter further in this section. This survey demonstrated a significant increase in cardiac, pulmonary, and coronary dosimetry following the initial introduction of 3D planning and routine cardiac contouring (Figure 6).

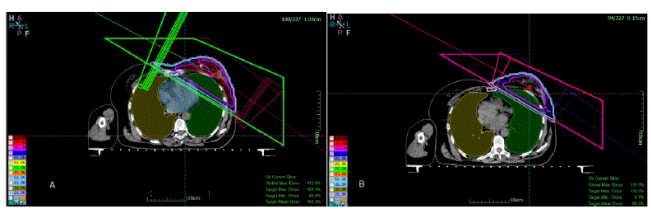

Figure 6: (A) Dose distribution from 6MV tangential irradiation. Dose distribution of conventional breast beams. Note the area of target, not covered by 95-percent isodose, when conventional beams are used, especially in medio-dorsal and latero-dorsal area of the breast. (B) Isodose distribution of 3DCRT tangential beams. This figure shows more accurate beam to target, with dose homogenicity. LAD coronary artery with a $1 \mathrm{~cm}$ margin is shown with light green.

In $2 \mathrm{D}$ method (SAD technique) which is considered as the conventional technique, patients' breasts were irradiated without any contouring. The only basis, on which conventional treatment of the breast is aligned, is patients' anatomy and landmarks. On the contrary, three-dimensional alignment is built up on the basis of multi-slice CT scan planes and with the help of treatment planning software (TPS) and advanced shielding. Computerized Tomography without contrast enhancement was ordered for each subject, in order to determine 3dimensional treatment plan. CT scan slices were of $1.5 \mathrm{~mm}$ thickness which is narrow enough to accomplish accurate reconstruction. The difference in dosimetrical indexes can be explained by the fact that in $2 \mathrm{D}$ method there is only slice (mainly the middle one) that is used for measuring doses to the organ. Hence that slice may not show maximal dose to which the organ is exposed, and therefore may not be the best slice to measure. On the contrary, in 3DCRT method we use multiple slices and treatment planning, resulting in much more accurate evaluating of the organs' dosimetry.

Only a few studies are available that evaluate cardiac dosimetry in routine clinical practice following changes from $2 \mathrm{D}$ to $3 \mathrm{D}$ planning. Findings of some studies were consistent with our results. In an old study by Taylor et al. mean heart dose averaged $5.1 \mathrm{~Gy}$, and mean left anterior descending coronary artery dose averaged 9.5 Gy during 2D (SAD) left-sided breast radiotherapy in 1950. This survey also revealed that all dosimetrical parameters were reduced substantially due to enhancement in techniques and maneuvers over time _"tangential irradiation changed considerably between the 1950s and the 1990s" as their exact words [16]. They also concluded that a strong linear correlation was found between the MHD and the mean heart dose and mean heart BED, and MHD is a reliable predictor of the mean heart dose and BED and gives an approximate estimate of the mean LAD coronary artery dose and BED. Another very important conclusion that Taylor et al. have made is that the mid-plane CT slice did not give a reliable assessment of cardiac irradiation. That is how we explain that almost all dosimetrical indexes are calculated lower in 2D (SAD) technique. This is due to the fact that the position of the slice showing the maximum area of irradiated heart (MHD) was determined by the location of the heart. In contrast, the position of the mid-plane slice was largely determined by the breast position. In one study carried out by Correa et al. patients with cardiac diagnostic test abnormalities had a larger median maximum heart distance (MHD) and central lungs distance (CLD of $2.6 \mathrm{~cm}$ vs. $2.2 \mathrm{~cm}, \mathrm{p}<0.05$ and MHD was $2.2 \mathrm{~cm}$ in both groups tangential RT). This study revealed that the incidence rate of cardiovascular disease was 39\% among symptomatic left-sided irradiated women, which was significantly higher than the predicted incidence of cardiovascular disease in the patient population. Although their study did not evaluate other RT parameters that we did [17]. For conventional radiation techniques, Hurkmans et al. reported that maximum heart distance (MHD) was a good parameter from which the normal tissue complication probability (NTCP) for excess late cardiac mortality could be estimated [18]. In another study carried out by Borger et al. they found no significant trend of increased risk with increasing maximum heart distance. Patients with a maximum heart distance greater than $3.0 \mathrm{~cm}$ seemed to have a higher risk of ischemic heart disease; however, patient numbers were small and did not reach statistical significance [19]. Graham et al. aimed to evaluate the cardiac dosimetry delivered in 2D and after routine 3D CT wholebreast radiotherapy planning including cardiac contouring, and to compare the two. Consistent with our results, findings by Graham et al. did not show a substantial reduction in cardiac or coronary irradiation despite the introduction of $3 \mathrm{D}$ planning and routine cardiac contouring. This may reflect inability to modify tangential field borders without affecting clinical target coverage or simply a lack of incorporation of additional contouring information into planning decisions. What came as their result could simply be because individual coronary dose data was not obtained but imputed from virtual simulation of treatment protocols, although their study used repeat individual CT planning to obtain an accurate calculation of dose in those patients treated prior to routine cardiac contouring [20]. They also showed that increase in the prescribed dose would result in a linear increase in organ dosimetry, with inferior LAD receiving the highest doses and the heart receiving a lower dose. Therefore if the LAD is the critical organ at risk with regard to the increased late mortality for left-sided breast radiotherapy, then perhaps the inferior LAD is the critical segment. If this is the case, then techniques such as deep inspiratory breath hold (DIBH) may make less significant differences to clinical outcomes than hoped for, since the LAD displacement and dose reduction are largely restricted to the proximal (superior) third with DIBH, and the proximal LAD usually receives low doses in any case[21]. Moreover, Marks et al. [22] and Das et al. [23] assessed the incidence of myocardial perfusion defects in around 70 women who received different heart doses from left-tangential radiotherapy. Another study on contoured vital organs carried out by Aznar et al. indicated that it is critical to assess the dose delivered to the whole heart as well as to the whole LAD when investigating the acceptability of a breast irradiation treatment. Assessing the dose to only one of these structures could lead to excessive heart irradiation and thereby increased risk of cardiac complications for breast cancer radiotherapy patients [24]. In a retrospective study on $2 \mathrm{D}$ treatment planning, carried out by Taylor and McGale, all RT plans for these patients were constructed before the era of routine three-dimensional (3D) computed tomography (CT)-based treatment planning; thus, all patient treatments were planned with fluoroscopic simulation using $2 \mathrm{D}$ radiographs. It can be perceived from their study that contemporary RT for early breast cancer may be associated with a small, but potentially avoidable, risk of cardiovascular morbidity that is associated with treatment technique. Also strong linear correlations between the MMD and the mean heart dose and LAD dose have been reported by Taylor et al. [25]. 
Citation: Hamid E, Ali A, Refagh S, Mehdi K, Alireza A, et al. (2017) Indexes of Cardiac and LAD Irradiation in Conventional 2-Dimensional (SAD) and 3-Dimensional Conformal Radiotherapy Techniques: A Dosimetrical Comparison. J Nucl Med Radiat Ther 8: 337. doi: $10.4172 / 2155-9619.1000337$

Page 6 of 6

Similarly designed studies revealed dosimetrical findings inconstant with our findings. For example in the study carried out by Graham et al. [20] changes in cardiac dosimetry associated with routine cardiac contouring had initially been minor and restricted to low-risk patients. A $15-\mathrm{mm}$ MMD reasonably represented a transition from low mean distal LAD doses to substantial doses. This lack of difference in dosimetry may be explained by their more advanced contouring methods. Storey et al. concluded that the risk of cardiovascular morbidity is associated with treatment technique and is potentially avoidable. Storey et al. evaluated dose-volume histograms (DVH) in left-sided radiation treatment with 2-dimensional (SAD) technique for early-stage breast cancer and found that the dose to the left anterior descending artery was strongly dependent on the central lungs distance, whereas the dose to the other coronary vessels was minimal [26].

The present study has quite a handful of limitations. Relatively small sample size, for instance, may have resulted in small slidings in dosimetrical prediction in each group of methods.

\section{Conclusion}

A $3 \mathrm{D}$ conforming radiotherapy had a significant dominance in almost all dosimetrical parameters we assessed. This finding can simply be explained by low efficacy in therapeutic doses applied in the conventional technique. Moreover, since there is no planning and contouring in conventional (SAD) technique, we assessed the middle slice of CT scan on dosimetrical parameters, in contrast to 3DCRT technique in which the slice that showed the vastest area of organ radiation was chosen for contouring and assessment. However, what seems and remains evident is that more enhanced equipments and techniques such as arm-suspension position are needed for contouring vital organs and radiation treatment, since the dose measured to be applied to these organs in $3 \mathrm{D}$ method was higher than what most studies have suggested as a threshold dose to organs.

\section{References}

1. McPherson K, Steel C, Dixon J (2000) Breast cancer-epidemiology, risk factors and genetics. BMJ 321: 624 .

2. Harirchi I (2004) Breast cancer in Iran: Results of a multi-center study. Asian pacific J cancer prevent 5: 24-27.

3. Manzouri L, Salehi R, Shariatpanahi S (2014) Prevalence of human papilloma virus among women with breast cancer since 2005-2009 in Isfahan. Advanced Biomed Res 3: 75.

4. Mousavi SM (2007) Breast cancer in Iran: An epidemiological review. The Breast J 13: 383-391.

5. Buchholz TA (2009) Radiation therapy for early-stage breast cancer after breast-conserving surgery. New Eng J Med 360: 63-70.

6. Harnett A (2010) Fewer fractions of adjuvant external beam radiotherapy for early breast cancer are safe and effective and can now be the standard of care: Why the UK's NICE accepts fewer fractions as the standard of care for adjuvant radiotherapy in early breast cancer. The Breast 19: 159-162.

7. Vinh-Hung V, Verschraegen C (2004) Breast-conserving surgery with or without radiotherapy: Pooled-analysis for risks of ipsilateral breast tumor recurrence and mortality. J National Cancer Ins 96: 115-121.
8. Darby SC (2013) Risk of ischemic heart disease in women after radiotherapy for breast cancer. New Eng J Med 368: 987-998.

9. Harris EE (2006) Late cardiac mortality and morbidity in early-stage breast cancer patients after breast-conservation treatment. J Clinical Oncol 24: 4100-4106.

10. Taylor CW (2007) Cardiac exposures in breast cancer radiotherapy: 1950s-1990s. International J Radiat Oncol Biol Phys 69: 1484-1495.

11. Chargari C (2011) Cardiac toxicity in breast cancer patients: From a fractional point of view to a global assessment. Cancer Treat Rev 37: 321-330.

12. Magné $\mathrm{N}$ (2010) Tomorrow's targeted therapies in breast cancer patients: What is the risk for increased radiation-induced cardiac toxicity? Critical Reviews Oncol Hematol 76: 186-195.

13. Roychoudhuri R (2007) Increased cardiovascular mortality more than fifteen years after radiotherapy for breast cancer: A population-based study. BMC cancer 7: 9.

14. Demirci S (2009) Radiation-induced cardiac toxicity after therapy for breast cancer: interaction between treatment era and follow-up duration. International J Radiat Oncol Biol Phys 73: 980-987.

15. Brunckhorst E, Gershkevitsh E, Ibbott G (2008) Commissioning of radiotherapy treatment planning systems. Testing for typical external beam treatment techniques. IAEA 1583: 1-67.

16. Taylor CW (2009) Cardiac doses from Swedish breast cancer radiotherapy since the 1950s. Radiotherapy Oncol 90: 127-135.

17. Correa CR (2008) Association between tangential beam treatment parameters and cardiac abnormalities after definitive radiation treatment for left-sided breast cancer. International J Radiati Oncol Biol Phys 72: 508-516.

18. Hurkmans CW (2000) Cardiac and lung complication probabilities after breast cancer irradiation. Radiotherapy Oncol 55: 145-151.

19. Borger JH (2007) Cardiotoxic effects of tangential breast irradiation in early breast cancer patients: The role of irradiated heart volume. International J Radiat Oncol Biol Phys 69: 1131-1138.

20. Graham P (2014) Cardiac dosimetry for adjuvant left-sided breast radiotherapy: Patterns with $2 \mathrm{D}$-versus $3 \mathrm{D}$-era planning and correlates of coronary dose with maximum depth of myocardial exposure. J Med Imag Radiat Oncol 58: 517-522.

21. Jarvis LA, Maxim PG, Horst K (2012) Deep inspiration breath hold reduces dose to the left ventricle and proximal left anterior descending artery during radiotherapy for left-sided breast cancers. J Cancer Therapy 3: 673 .

22. Marks LB (2005) The incidence and functional consequences of RTassociated cardiac perfusion defects. Internat J Radiat Oncol Biol Physi 63: 214-223.

23. Das SK (2005) Predicting radiotherapy-induced cardiac perfusion defects. Med Phys 32: 19-27.

24. Aznar MC (2014) Evaluation of dose to cardiac structures during breast irradiation. The British J Radiol 84: 743-746.

25. Taylor C (2009) Estimating cardiac exposure from breast cancer radiotherapy in clinical practice. International J Radiat Oncol Biol Phys 73: $1061-1068$.

26. Storey MR (2000) Coronary artery dosimetry in intact left breast irradiation. Cancer J (Sudbury, Mass) 7: 492-497. 